\title{
Managing a male golfers low back and sacroiliac joint pain using manual therapy and therapeutic exercises to avoid surgery and continue scratch level play.
}

\author{
Stephen A Black* \\ Department of Rehabilitation Sciences, Marieb College of Health \& Human Services, Florida Gulf Coast University, \\ 10501 FGCU Blvd South, Fort Myers, FL 33965, USA
}

\begin{abstract}
Purpose: The purpose of this case study is to demonstrate a unique way of working with low handicap golfers looking to avoid surgery after two years of low back/sacroiliac pain (LBP/SIJP) and injury. This study used all-encompassing treatment sessions each time the patient was seen, including lengthening, strengthening and creating power with less stress on the LB/SIJ region. The treatment looked at decreasing muscle and structural imbalance and engaging all of the muscles when making a swing.

Client description: Patient is a 71 year-old man with a 14 month "bad" back with history of pain. The pain was so debilitating that he went from daily play to a three time a week play. His play itself also decreased from a +2 handicap to a -4 handicap. The patient complains that he suffers from constant pain and has decreased all functional and recreational activities.

Intervention: Patient was seen 2-3 times a week for eight and half months. There was a three-month stop when the patient returned north for the summer and was healing from an arm injury. The patient completed treatments targeted at maintaining a level pelvis, increasing mobility of the lumbar spine, and strength training all surrounding core, gluteal and lower extremity muscles. Muscles Energy Techniques (MET) were used to align his pelvis. Stabilization exercises and strengthening exercises that could be completed in the golf position were done in order to create proper length tension relationships as well as kinesthetic awareness of the body during the swing.

Balance and proprioception was also worked on every session with a push/pull concept in order to decrease muscle imbalances and increase coordination from past months of pain and injury.

Measures and Outcomes: All measurements and subjective and objective measures were covered over 8 months. After the second treatment patient's pain level and ability to move independently was improved significantly. IR and ER of the hips took the longest to achieve but this measurement directly affected the pelvis alignment and patient's subjective comments on the ability to get through the ball during the swing. His pain and tone levels continued to go down throughout treatment and he is now without pain during and following play.

Implications: "Once a bad back, always a bad back" appears to be a proper theme for athletes who wish to continue to play at the same level or better without surgery or medicine intervention. Patients who are willing to work on their body with manual and physical strength training can keep most symptoms in control and still have improvement in play. But it requires daily exercise and manual stretching. Pain was decreased and function was returned in about 25 days, but whenever the patient stopped his exercises and stretching (within the first 5 months of treatment), symptoms would return. He is now able to play and travel with less routine, but still needs at least 1 time a week for core strengthening and stretching. Golf mechanics have been resumed because there is a lack of pain and all the muscles that should be used are now turned on with the specific strength routine.
\end{abstract}

\section{Introduction}

LBP and SIJ dysfunction are two of the most common golf related symptoms representing up to $52 \%$ of all musculoskeletal complaints/injuries [1]. Because LBP is already costing the nation over $\$ 50$ billion dollars a year to treat, it is vital to come up with ways to prevent or decrease LBP and SIJ dysfunction among golfers so that there is not more money spent nationally to deal with these pathologies [2].
Injury to a specific area still affects the entire body. Proprioception (the unconscious knowledge of where the body is in space) can be lost if an injury is sustained. The longer the patient is not playing, the greater the loss of the proprioception and kinesthetic awareness. In addition, coordination will also be lost and timing of the mechanics will be off, decreasing patients overall ability to play and score well. Once a golfer shortens his stroke and loses their timing, the harder it is to return to same level play. 
Citation: Black SA. Managing a male golfers low back and sacroiliac joint pain using manual therapy and therapeutic exercises to avoid surgery and continue scratch level play. J Phys Ther Sports Med 2018;2(1):33-39.

Back pain is common among golfers due to the fact that the golf swing requires many complex movement patterns with coordinated muscle contractions and nerves firing in proper sequence [3]. With the complex movements needed to complete a full golf swing, one must understand that back injury is rarely the primary cause of LBP. Dr. Greg Rose has researched why low back injuries are not the primary cause of pain but rather secondary to the massive amount of rotational mobility that is needed in the thoracic spine and hip region. The lumbar spine actually only flexes and extends and is not created to rotate at the facet joints. But at times the lumbar spine is forced to be the primary rotator due to the lack of mobility in the thoracic spine and hip region. In addition, golfers who lack ankle and shoulder mobility also increase lumbar strain [3]. So in reality, injuries to the spine are caused from limitation of movement of other parts of the body and these areas must be addressed if a patient wishes to decrease the risk for re-injury [4].

It is vital that the swing mechanics are reviewed and corrected in order to minimize lumbar pathologies and overuse injuries. The most common swing faults that are seen are reverse spine angle, s-posture, and hanging back [4]. These faults put undue stress on the lumbar spine and SI joints. There are many different swing mechanics, but we are not looking at that, rather we are looking at the tightness and restriction of the muscles in the body that cause the LBP and SIJ dysfunction, and how to remedy these limitations so players can continue to play at their current golf level [1].

Biomechanics of the human body affect how muscles can strengthen and change with training. For athletes it is important for muscles to have the proper length tension relationship in order to perform at an elite level. It has been researched that golfers suffer from a muscle imbalance between lead and trail side of the body, as well as range of motion in the joints.

\section{Case Description}

\section{History}

On arrival to therapy this 71 year old male patient was convinced that he was destined to have a fusion of the L4-L5S1 bilaterally. He was defeated, in pain and discouraged since the bilateral epidural injection that he had 10 days ago was unsuccessful. The patient had been suffering from "bad" pain for the past 14 months and his play of game was suffering. Patient complained of inability to sleep more than 4 hours without waking from pain and having a 6-8/10 pain with all activities. Patient was under better control as long as he took anti-inflammatory medicines. Patient presented with an unlevel pelvis; supine to long sit test was completed and was positive. Left leg appeared longer in supine and shortened upon long sit. MET was used to re-align the pelvis. Next the patient performed the Selective Functional Movement Assessment (SFMA). Eighty percent of all the completed tests were graded as dysfunctional with pain. The most important test that was completed on evaluation day (and every other treatment day) was IR and ER of the hip in supine and standing. Supine revealed limitations bilaterally 20-22 degrees both ways, which was possibly the main cause of patients LBP and SIJP. Standing test revealed pain in both hips regardless of which leg was in high step position or standing and in both directions. Hip windshield wipers were very challenging and painful as well.

\section{Findings}

It was apparent that two years of pain and injury had altered which muscles fired and worked with the swing. As we continued to work on strength and length of the patient's body, we figured out that new injuries and lack of playing caused a "lazy" swing. With hours of training, strengthening, stretching and manually correcting the length tension relationship of the core, lumbar and gluteal muscles, the patient returned to playing pain free. It was learned that the patient must continue with strengthening and stretching weekly in order to maintain his current level. Patient has not needed or had another epidural injection and has not returned to the orthopedic surgeon for any discussion of fusion of the lumbar spine. Muscle tissue is now normalized and patient is able to complete new exercises at different intensities with no detriment to his overall play. Patient has also returned to scratch play according to GIN handicap scoring, with a goal to return to his +2 .

\section{Intervention}

The patient was seen 2-3 times a week for 8.5 months with a 3month stop with HEP. After all of the evaluation tests were completed and information was known about what was tight and what was weak, the process began.

It is important for the patient to understand what is going on in the body. The lack of proprioceptive knowledge of the body is potentially the worst component in a golfer. With an injury and a general lack of body knowledge there is an increased risk for more secondary injuries. Therefore all sessions conducted had some form of re-education and engagement of muscles working on proprioception, balance and kinesthetic awareness.

SIJ level was assessed each time pre and post treatment session in order to maintain proper alignment for strength training and relief of pain. Patient was taken through passive pelvic tilt movements in quadruped to re-learn the motion as well as increase mobility of the lumbar spine, while stretching the anterior capsule of the hips. LB mobility was further assessed in each treatment with PA glides to make sure they were smooth and decreasing in pain. Lower extremity flexibility focused on IR and ER of the hips and was done manually to avoid patient using other muscles. IR and ER limitation in golfers can be [5]. When patients lack 0-45 degrees of motion they are at increased risk for LBP and arthritic changes in the hip joints. This is very damaging to the lead hip in a golfer.

Each session included exercises and manual work discussed above but in varying order so that there was no muscle memory. Patient saw an increase in function within about 30 days. Patient had less pain and more stability and strength to maintain position when swinging the club. The detailed 
exercise routine is attached listing all exercises that were completed throughout the 8 months of care (Table 1).

At the end of the season when the patient was about to gear up for a senior tour event, he had an unrelated golf injury where he tore his right brachioradialis muscles and tendons picking up a piece of luggage. This did decrease his ability to play but we continued with the lower body exercises and the patient did play in the event. Once the event was over the patient took some time off to heal the arm. This caused a significant change in his overall play when he returned to golf 2.5 months later. As documented by Dr. Greg Rose, golfers that have injury to the right arm often increase the stress to the opposing hip. When the patient returned to play he was demonstrating a "lazy" swing where he was unable to get his arms into position. He was unable to supinate his arms and bring the club back with the face of the club in the correct position (it was closed) therefore he was not using his lead hip to push through the swing. This left a lot of the strength and power out of the swing, causing the injured arm to work harder and the swing to almost get "stuck" so the patient was unable to get through the swing. This caused more strain and pain in the low back region. Once the lazy swing was seen, a new focus was created on rotational push exercises and more engagement of the quadriceps. Patient showed very week quadriceps strength $3+/ 5$ with unilateral lift step test of the quadriceps. This weakness deceased the patient's ability to maintain a good address position and stay down in the swing with power through the adductor of the lead hip.

Multiple new exercises were completed to assist in reeducating the lead hip adductor muscles to contract and assist in pulling the right arm through the swing. Punch turns with the hip leading were also completed and this allowed the patient to feel the power of those muscles again. Squat chop turns were used to elongate and create coordinated movements to strengthen the quadriceps, adductors and quadratus muscles bilaterally. Multiple exercises were completed, and no two workouts were the same. The difference with this type of treatment is to work the body in so many different angles that all muscles are reactivated during the swing and play. All sessions ended with manual work to lengthen the muscle tissues in the legs, gluteal and lumbar spine (Table 2).

Table 1. Manual work that was completed.

\begin{tabular}{|c|c|}
\hline Manual work completed & Body position/purpose \\
\hline MET & To correct the pelvis position in supine \\
\hline PA glides to the spine & Prone to thoracic and lumbar spine \\
\hline Sacral glides & Prone and side lying rotational movement of the sacrum/torsional movement \\
\hline Hamstring contract relax stretch & Supine \\
\hline Tibial/fibula glide to release the peroneal nerve & $\begin{array}{l}\text { Supine with knee bent anterior and posterior glide of the fibula on the tibia to } \\
\text { release the nerve entrapment of the peroneal/tibial nerve. }\end{array}$ \\
\hline ITB stretch and manual release & $\begin{array}{l}\text { Side lying and supine to manually release the tissues and stretch across the } \\
\text { body }\end{array}$ \\
\hline Rib mobilization with quadratus lumbourm stretch & Side lying with top arm reaching over the head \\
\hline Manual stretch with manual massage to the gluteal muscles & Supine and side lying with IR and ER of the hip \\
\hline Nerve glides to the sciatic nerve and peroneal nerve & $\begin{array}{l}\text { Supine with leg extended and rotated across the body with inversion stretch. } \\
\text { Knee extended and flexed with inversion }\end{array}$ \\
\hline Laser therapy class 4 cone & 12 watts to the SIJ B \\
\hline Sacral glides & Prone \\
\hline Hip flexor stretch & Side lying and prone \\
\hline ITB manual stretch and manual work & Supine \\
\hline Contract relax hamstring stretch & Supine \\
\hline Open book stretch bilaterally with full arm extension & Supine with manual rotation of the lumbar and lower legs \\
\hline Trigger point release of the hip flexors with active motion, gluteal mm & Prone, and supine and side lying \\
\hline Manual stretch on the table of upper and lower body in opposite ways & Supine and seated \\
\hline Golf stretch to top of back swing with passive stretch at end range & Standing in golf position \\
\hline Standing hamstring stretch with active body rotation & $\begin{array}{l}\text { Torso rotates to right and left with each leg flexed and on a step with toes } \\
\text { pulled toward nose }\end{array}$ \\
\hline
\end{tabular}


Citation: Black SA. Managing a male golfers low back and sacroiliac joint pain using manual therapy and therapeutic exercises to avoid surgery and continue scratch level play. J Phys Ther Sports Med 2018;2(1):33-39.

Posterior shoulder capsule stretch in golf position with passive end range stretch

Golf position in standing pull each arm across body and go into BS and FT position Triceps stretch in standing and sitting posture with elbow pointing up and over pressure Standing and sitting
added

Golf position standing

ER of the shoulders in golf address position with BS and FT

Maintain head position over the ball and in golf position stretch to BS and FT

Hands behind back rotation of the torso in golf position to stretch the ribs

Quadruped right hand on head and thread the needle and rotate back so elbow points to the ceiling

Pudendel nerve glide

Quadruped and go both ways with over pressure at the rib cage and shoulders

Prone with knee flexed and adducted

Latissimus stretch in half squat on the wall and in supine elbows kept close

Partial squat on wall with back against wall or supine with LB kept flat on the mat

Table 2. Exercises that were completed.

\section{Exercises completed}

Cat and Camel (pelvic tilt test) quadruped

Scorpion

Quadruped diagonal elbow to knee touch and hold

ITB standing stretching with side bend reach

Piriformis stretch

Single limb stance with dynamic balance

Backswing stretch/balance follow through

Bridges one leg/double leg

Bridge figure 8

Bridge routine on thera ball

Shot putt ex with $5 \#$ small ball

Squat chop diagonal push away and above

Bosu Prance

Lay on Thera Ball in bridge position

\section{Description of exercise}

Quadruped: create arch and pelvic tilt of the pelvis and lumbar spine; $5 x$ each direction with a 2 second hold each way.

Prone: kick right heel up to ceiling and than over the left side of the body trying to reach your right heel to the floor. Go both ways. Try to keep your upper body on the ground.

Quadruped hold right arm out extended and left leg extended out. Slowly and with control bring right elbow to left knee under the abs. The return out to straight pointing in opposite directions, $5 x$ each side.

Place all weight on right leg, cross left leg in front. Stick butt out behind you and then over to the right side. While reaching right hand to the left over your head. No bounce just a hold.

Figure 4 stretches in supine. Cross right foot over left knee and pull left knee to the chest.

Stand on the right leg with knee soft and then catch a ball and move your head to watch the moving target to catch ball

Go into address position: place $85 \%$ of your weight on the right leg left toe behind you. Rotate body to the top of the back swing and hold. Keep weight on the inside of the right foot. Place weight on the left leg and complete the follow through.

Supine: feet flat and lift the butt up and off the table both legs push. 10x then complete same action but with toes raised off the table and only push off of the heels. 10x then cross right foot over left knee and lift butt off table using only the left leg. 10x switch legs.

Bridge up on both legs. Hold and drop the right hip down and then bring back to neutral and drop the left hip. 5x each way.

Use large thera ball place heels on top of the ball complete the series of butt lifts: Knees straight, knees bent to 90 degrees and push only through heels, place legs adductor contraction and then lift, and do roll outs so legs start extended and then keep butt up while you pull both heels toward your butt and then return to straight.

Go into a squat or start of shot putt position: with arm down and ball held at shoulder height. Use the power from your legs and hips to push up and turn to the left. Extend the right arm across your body and up the sky 10x. Try to do both ways.

Hold ball at waist height and then drop down into a squat with ball over right knee and then with power stand tall and turn to the left pushing the ball from the right knee across the body and above the shoulders to full elbow extension toward the target.

Lay over the BOSU with butt close to the ground and low back supported by BOSU. Maintain a flat back try to get body flat across BOSU and rest legs up on their toes and pick each leg up controlling the body and core while you prance with each light leg step.

Have the ball support your head and back. Butt and legs are off of the ball and held in a perfect table top (do not drop the butt) hold a $5 \#$ small ball in both hands held above your head. Stomach stays tight maintain pelvis position to be flat and straight to the 
ceiling rotate the upper body on the ball to the right and upper body on the ball to the right and then left. The head can turn with you. Try to keep gluteal $\mathrm{mm}$ tight and flat.

Lunge step walk with rotation of large ball

Thera Ball Golf swings

Plank series

Gluteal exercises set

Hip set standing

Hip abduction and adduction on machines

Hip abduction machine: Complete IR and ER ex

Bosu step drills

Suma squat with weights in front

Hamstring raised and extended out stretch

Step ups

Hip hikes

Heisman move

Standing hip circles on gliders

Standing lumbar spine rotation

TRX golf stretch

TRX decompression on lumbar spine and pelvis

All upper body weights on balance discs

Hip flexor stretch active

Push/pull on kinesis

Lat pull down on knees on Bosu

Rotator cuff series with bands
Walking long lunges with control of rotation from the torso with head kept centered. Rotate the ball and torso to the right and left with each held lunge step.

In golf position hold large thera ball and complete a slow swing

Plank hold on elbows 30-60 seconds, Plank hold with elbows extended 30-60 seconds Plank march: Start on elbows and extend right arm first then left and then lower back down on to each elbow for 5 on the right lead and than 5 on left lead.

Side lying: top leg extended toe turned down and lift and lower with no core movement (10x). Bend both knees to 90 degrees extend top leg. Turn top foot so toe points down and lift with no body motion (5x). Both knees at 90 degrees and in front. Keep body still and feet touching and open the knees as wide as you can with no other motion.

Hip extension, hip abduction (with no lean) hip flexion and hip flexion with ER to open the hip. Keep pelvis level and facing fwd.

20 reps slow with a hold at each end range.

Place stomach on the back of the machine and knees on the seat of the hip abduction machine. Weight down to $10 \#$, place shins inside mounts and try to open the shins. Try to keep back flat and stomach against the back of chair.

Step overs for lateral movement with control. Step fwd. with opposite knee lift and hold. Stand on BOSU and step backwards with control.

Maintain proper form: wide stance with toes out. Squat down with weights in between the legs, do not bend at the waist and keep your butt under your body. Once completed 10 reps hold in down position and complete heel raises for 10 .

With leg extended and raised, rotate to the right and then reach for shin and hold. Then return to center and turn to the left and reach for the shin.

Slow step ups with the leg on the step doing all the work. Maintain strength and balance with step up.

Have right leg hanging off the step and hike the hip with no other body motion and then lower the hip in straight plane feel the separation from the top of the hip and the ribs.

Standing weighted ball in both hands over the right shoulder. Bring the ball to the raising left knee and touch to the outside of the leg knee. Go both ways.

Stand in golf position. Bilateral hand support as needed. Feet are on gliders and complete full clockwise and counter clockwise circles with each leg.

Lean onto a bar so spine is in address position. Open right hip and then bring right knee up and across to left elbow that is resting the bar. Complete 5-10x and then do the opposite side.

Stand with hands on TRX straps in address position open arms and go all the way into the back swing and hold to stretch and then go into the full follow through.

Hold onto TRX straps with arms flexed, squat all the way down to the ground holding yourself up with arms and legs. Let pelvis be heavy and hold for stretch.

Stand on balance discs in address position: complete bicep curls, triceps kickbacks, shoulder windshield wipers and shoulder extension bilaterally, does not lose address position.

Standing hold onto a bar: place right knee and shin on the thera ball and push back the right hip and wait for the stretch in the hip. Keep pelvis level and hold.

With both arms individually stand in lunge position and push right arm into chest press for 15 and then turn around legs in same position and pull back the weight with the shoulder blade. Stomach is held tight.

Balance on Bosu on your knees and complete Lat pull down to the front of the chest at the sternum level, make sure the arms are wide and in control. Lean slightly back and maintain balance and pull with strength (smooth and control).

Frog leg crunch to get hip rotated and lifted, oblique crunch with opposing knee to shoulder, side leg and upper body lift, legs extended and upper body side climb the rope with extend the arms and reach and lift.

Standing in golf position: IR and ER of the shoulders at waist level and at 90/90 level. 
Citation: Black SA. Managing a male golfers low back and sacroiliac joint pain using manual therapy and therapeutic exercises to avoid surgery and continue scratch level play. J Phys Ther Sports Med 2018;2(1):33-39.

Concentric strength machines

Full body circuit for legs: leg press wide with toes out and close with a ball between the legs, leg extension, leg curls, hip abd and add.

Standing torso rotation: with free motion machine In athletic stance rotate to the right and left with no head turn and no pelvis turn.

Table 3. Measures and outcomes of case study.

\begin{tabular}{|c|c|c|}
\hline Tests & IE Results & Final Results \\
\hline FABERS & $+B$ for all $3 \mathrm{MM}$ & B for hip flexor intermittently \\
\hline Pelvis Alignment & Anteriorly rotated on left side & Level pelvis \\
\hline Oswestry/pain scale & $46 \%$ disabled secondary to LBP & $2 \%$ of disability secondary to LBP \\
\hline Medication for pain & +4 Aleve a day & +2 Aleve a week \\
\hline Quadruped pelvis tilt test & +Impossible to complete & Can control and complete as ex \\
\hline ADLS & Unable to do anything more than ADLS & $\begin{array}{l}\text { Able to walk and work out on top of golf } \\
\text { and ADLS }\end{array}$ \\
\hline Practice times & $1-2 \mathrm{hrs}$ a week & 7-8 hrs a week \\
\hline Playing & 2-3 times a week & 7 days a week \\
\hline Hamstring length & 40 Degrees bilaterally with pain & $\begin{array}{l}80 \text { Degrees } B \text { and with end range } \\
\text { discomfort }\end{array}$ \\
\hline Manual MM tone & For all MM surrounding the pelvis and LB & Minimal tone intermittently \\
\hline IR/ER of hip supine & 22 Degrees bilaterally & L IR: 38 ER: 46 R IR: 34 ER: 48 \\
\hline SFMA & $\begin{array}{l}6 \text { functional no pain } 11 \text { dysfunctional pain } 3 \text { dysfunctional } \\
\text { no pain }\end{array}$ & $\begin{array}{l}15 \text { functional no pain } 2 \text { dysfunctional } \\
\text { pain } 4 \text { dysfunctional no pain }\end{array}$ \\
\hline
\end{tabular}

All measurements and subjective and objective measures over the 8 months are outlined in Table 3 in above section. After the second treatment patient's pain level and ability to move independently was improved significantly. IR and ER of the hips took the longest to achieve but this measurement directly affected the pelvis alignment and patient's subjective comments on the ability to get through the ball during the swing. His IR/ ER on initial evaluation was 20-22 degrees and now is 38-40 bilaterally. The patient's pain was reduced $50 \%$ within 2 weeks of working on muscle length and tone. His pain and tone levels continued to go down throughout treatment and he is now without pain during and following play. Muscle engagement or contractions now decrease any new pain that arrives following play. Isometric contractions complete during play and in address position have assisted with kinesthetic awareness and feeling of getting through the ball without getting "stuck" in the back swing. Patient is now able to complete the pelvic tilt test in quadruped, standing and in address position, which is a great kinesthetic awareness return post injury. This patient has had an excellent outcome that came from hard work. He must continue with manual stretching and massage, as well as strength training all angles of the body, in order to maintain this level of play. It is not without extra time and fees that this pain will stay away. This is his new routine that he must continue to maintain his level of scratch level play.

\section{Discussion}

The purpose of this case study was to look at how we can better manage our patient's pain and disabilities while avoiding injections and surgeries and allowing them continuation of play. It was important to first educate the patient on life after spinal fusion surgery compared to golf play without surgery. Spinal fusion is a significant surgery that would have limited the patient from swinging a club (not actually playing) for 8-12 months post-surgery [1]. To an athlete this can be seen as a death sentence. So it was very important to educate the patient on the pros and cons of both directions for treating his back pain, and to get the commitment to treatment that was needed to avoid this surgery and continue to play.

All evaluations start with assessing the patient to determine their needs and limitations to make sure they are safe to resume play and to participate in a full-scale multi-dimensional exercise and manual therapy program. In this specific case our goals originally worked to decrease the patient's pain and determine the current level of function and ability of this patient. The patients MRI have shown us discs and boney segments with arthritic changes, but it was the overall muscle imbalance and weakness that caused the lack of spinal and sacral mobility. This led to a decrease in the patient's ability to move in the correct biomechanical path and resulted in his pain. This was evident after we broke down the swing patterns of the body and tested out each of the body segments for balance, strength, stability and endurance. It was then that we realized how limited this patient was in terms of what he was 
requiring his body to do. The Selective Functional Movement Assessment (SFMA) was very helpful in breaking down each aspect of dysfunction in the swing and his active movements, so we could see which aspect of the movement was limited and if it was structural or muscular. As stated in the Nourbakhsh study of the relationship between "mechanical factors and incidence of low back pain," they concluded that muscle weakness and decreased muscle endurance was associated strongly with low back pain and that the actual structural factors like lumbar lordosis, pelvic tilt and length of muscles of hips and abdomen did not associate with LBP [6]. This is what was seen in my case as well. The actual arthritic changes and disc herniation were not the specific cause of LBP but rather a result of poor mechanics that were created by weakness and tightness of muscles and capsules when the body tried to swing.

I feel that it is necessary to have a combination of both strengthening and manual work to lengthen the muscles. This combination allows for better body mechanics and decreases the risk of increased force on joints over years of play. Fitness and manual programs were created to strengthen, educate and lengthen all aspects of the body and mind. Golf is a full body sport that adds significant force through the spine and rotational torsion to multiple joints. This effort requires golfers to participate in a multi-discipline fitness and manual program in order to keep the joints and supporting tissues healthy.

\section{References}

1. Gluck GS, Bendo JA, Spivak JM. The lumbar spine and low back pain in golf: a literature review of swing biomechanics and injury prevention. Spine J 2008;8(5): 778-88.
2. McHardy A, Pollard H. Lower back pain in golfers: a review of the literature. J Chiropr Med 2005;4(3):135-43.

3. Rose G. The golfer's guide to lower back pain. Available from http://www.mytpi.com/articles/health/the golfer's guide to lower back pain/ Accessed on: July 27, 2017.

4. Rose G. Back to Golf: Important Considerations for Golfers Undergoing Rehab Following Back. Available from http:// www.mytpi.com/articles/health/

back_to_golf_important_considerations_for_golfers_under going_rehab_following_back_surgery/ Accessed on: July 27, 2017.

5. Murray E, Birley E, Twycross-Lewis R, et al. The relationship between hip rotation range of movement and low back pain prevalence in amateur golfers: an observational study. Phys Ther Sport 2009;10(4):131-5.

6. Nourbakhsh MR, Arab AM. Relationship between mechanical factors and incidence of low back pain. J Orthop Sports Phys Ther 2002;32(9):447-60.

\section{*Correspondence to:}

Dr. Stephen A. Black

Department of Rehabilitation Sciences

Marieb College of Health \& Human Services

Florida Gulf Coast University

Fort Myers, FL 33965, USA

Tel: $239-590-1281$

E-mail: sblack@fgcu.edu 